\title{
Quantum Current Operators-I Zeros and Poles of Quantum Current Operators and the Condition of Quantum Integrability
}

\author{
By
}

\author{
Jintai Ding* and Tetsuji Miwa*
}

\begin{abstract}
For the current realization of the affine quantum groups, a simple comultiplication for the quantum current operators was given by Drinfeld. With this comultiplication, we study the zeros and poles of the quantum current operators and present a condition of integrability on the quantum current operators of $U_{q}(\hat{\mathfrak{s} l}(2))$, which is a deformation of the corresponding condition for $\hat{s} l(2)$. We also present the results about the zeros and poles of the quantum current operators of $U_{q}(\hat{\mathfrak{s} l}(n))$.
\end{abstract}

\section{§1. Introduction}

For any integrable highest weight module of $\hat{\mathfrak{s} l}(2)$ of level $k$, the current operators $e(z)$ and $f(z)$ satisfy the following relations.:

$$
e(z)^{k+1}=f(z)^{k+1}=0,
$$

which we call the condition of integrability [LP].

Quantum group was discovered by Drinfeld [Dr1] and Jimbo [J1] as a new structure in both mathematics and physics. The definition of a quantum group is given by the basic generators and the relations based on the data coming from the corresponding Cartan matrix. However for the case of quantum affine algebras, Drinfeld presented a different formulation of affine quantum groups with generators in the form of current operators [Dr2], which, for the case of $U_{q}(\hat{\mathfrak{s} l}(2))$, give us the quantized current operators corresponding to $e(z)$ and $f(z)$ of $\hat{\mathfrak{s} l}(2)$. One natural problem is to find out if it is possible to find a condition of integrability for the quantum current operators, which is a deformation of the classical condition of integrability above. The non-commutativity

Communicated by M. Kashiwara, September 10, 1996.

1991 Math. Subject Classification(s) : 17B37

*RIMS, Kyoto University, Kyoto, 606-01, Japan. 
of those quantum current operators makes the problem much more difficult than the classical case. To solve this problem, we need to use a new comultiplication given by Drinfeld, which we call Drinfeld comultiplication.

For the new formulation of affine quantum group, Drinfeld proposed another comultiplication formula $[\mathrm{DF}],[\mathrm{DI}]$ based on such a formulation. The fundamental feature of this comultiplication is its simplicity, while the comultiplication formula induced from the usual comultiplication can not be written in a closed form with those current operators. With this comultiplication, we are able to study the zeros and poles of quantum current operators for integrable modules. Our main results are Theorem 7 and 8 which state that on any level $k$ integrable module of $U_{q}(\hat{\mathfrak{s} l}(2))$ the matrix coefficients of $x^{+}\left(z_{1}\right) x^{+}\left(z_{2}\right) \cdots \cdot x^{+}\left(z_{k+1}\right)$ have zero at $z_{2} / z_{1}=z_{3} / z_{2}=\ldots=z_{k+1} / z_{k}=q^{2}$, and those of $x^{-}\left(z_{1}\right) x^{-}\left(z_{2}\right) \cdots x^{-}\left(z_{k+1}\right)$ have zero at $z_{1} / z_{2}=z_{2} / z_{3}=\ldots=z_{k} / z_{k+1}=q^{2}$, where $x^{+}(z)$ and $x^{-}(z)$ are the quantized current operators of $U_{q}(\hat{\mathfrak{S} l}(2))$ corresponding to $e(z)$ and $f(z)$ of $\hat{\mathfrak{s} l}(2)$, respectively. We first study the case of the fundamental representations of level 1 for $U_{q}(\hat{\mathfrak{g} l}(2))$. Frenkel and Jing used bosonized vertex operators to construct explicit realizations of those representations. It is clear that all the integrable modules can be derived from certain tensors of fundamental representations. Therefore, with the Drinfeld comultiplication, we can derive the poles and zeros of the quantum currents, which naturally leads to a condition of integrability for the quantum currents of $U_{q}(\hat{\mathfrak{s} l}(2))$. At the end, we present the corresponding results for $U_{q}(\hat{\hat{s} l}(n))$.

\$2。

For the case of affine quantum groups, Drinfeld gave a realization of those algebras in terms of operators in the form of current [Dr2]. We will first present such a realization for the case of $U_{q}(\hat{\mathfrak{s} l}(n))$.

Let $A=\left(a_{i j}\right)$ be the Cartan matrix of type $A_{n-1}$.

Definition 1. The algebra $U_{q}(\hat{\mathfrak{S l}}(n))$ is an associative algebra with unit 1 and the generators : $\varphi_{i}(-m), \psi_{i}(m), x_{i}^{ \pm}(l)$, for $i=i, \cdots, n-1, l \in \mathbb{Z}$ and $m \in$ $\mathbf{Z}_{\geq 0}$ and a central element $c$. Let $z$ be a formal variable and $x_{i}^{ \pm}(z)=\Sigma_{l \in \mathbf{Z}}$ $x_{i}^{ \pm}(l) z^{-l}, \varphi_{i}(z)=\sum_{m \in-\mathbf{Z}_{\geq 0}} \varphi_{i}(m) z^{-m}$ and $\psi_{i}(z)=\sum_{m \in \mathbf{Z}_{\geq 0}} \psi_{i}(m) z^{-m}$. In terms of the formal variables, the defining relations are

$$
\begin{aligned}
& \varphi_{i}(z) \varphi_{j}(w)=\varphi_{j}(w) \varphi_{i}(z) \\
& \psi_{i}(z) \psi_{j}(w)=\psi_{j}(w) \psi_{i}(z) \\
& \varphi_{i}(z) \psi_{j}(w) \varphi_{i}(z)^{-1} \psi_{j}(w)^{-1}=\frac{g_{i j}\left(z / w q^{-c}\right)}{g_{i j}\left(z / w q^{c}\right)}
\end{aligned}
$$




$$
\begin{aligned}
& \varphi_{i}(z) x_{j}^{ \pm}(w) \varphi_{i}(z)^{-1}=g_{i j}\left(z / w q^{\mp \frac{1}{2} c}\right)^{ \pm 1} x_{j}^{ \pm}(w), \\
& \phi_{i}(z) x_{j}^{ \pm}(w) \phi_{i}(z)^{-1}=g_{i j}\left(w / z q^{\mp \frac{1}{2} c}\right)^{\mp 1} x_{j}^{ \pm}(w), \\
& {\left[x_{i}^{+}(z), x_{j}^{-}(w)\right]=\frac{\delta_{i, j} \cdot}{q-q^{-1}}\left\{\delta\left(z / w q^{-c}\right) \phi_{i}\left(w q^{\frac{1}{2} c}\right)-\delta\left(z / w q^{c}\right) \varphi_{i}\left(z q^{\frac{1}{2} c}\right)\right\},} \\
& \left(z-q^{ \pm a_{i j}} w\right) x_{i}^{ \pm}(z) x_{j}^{ \pm}(w)=\left(q^{ \pm a_{i j}} z-w\right) x_{j}^{ \pm}(w) x_{i}^{ \pm}(z), \\
& {\left[x_{i}^{ \pm}(z), x_{j}^{ \pm}(w)\right]=0 \text { for } a_{i j}=0,} \\
& x_{i}^{ \pm}\left(z_{1}\right) x_{i}^{ \pm}\left(z_{2}\right) x_{j}^{ \pm}(w)-\left(q+q^{-1}\right) x_{i}^{ \pm}\left(z_{1}\right) x_{j}^{ \pm}(w) x_{i}^{ \pm}\left(z_{2}\right)+x_{j}^{ \pm}(w) x_{i}^{ \pm}\left(z_{1}\right) x_{i}^{ \pm}\left(z_{2}\right) \\
& \quad+\left\{z_{1} \longleftrightarrow z_{2}\right\}=0, \text { for } a_{i j}=-1
\end{aligned}
$$

where

$$
\delta(z)=\sum_{k \in \mathbf{Z}} z^{k}, g_{i j}(z)=\frac{q^{a_{i j}} z-1}{z-q^{a_{i j}}} \text { about } z=0
$$

In [Dr3], Drinfeld only gave the formulation of the algebra. If we extend the usual comultiplication to those current operators, the result would be a very complicated formula which can not be written in a closed form with only those current operators. However, Drinfeld also gave the Hopf algebra structure for such a formulation in an unpublished note.

Theorem 2. The algebra $U_{q}(\hat{\mathfrak{s} l}(n))$ has a Hopf algebra structure, which is given by the following formulae.

Coproduct $\triangle$

$$
\begin{aligned}
& \Delta\left(q^{\frac{c}{2}}\right)=q^{\frac{c}{2}} \otimes q^{\frac{c}{2}}, \\
& \Delta\left(x_{i}^{+}(z)\right)=x_{i}^{+}(z) \otimes 1+\varphi_{i}\left(z q^{\frac{c_{1}}{2}}\right) \otimes x_{i}^{+}\left(z q^{c_{1}}\right), \\
& \Delta\left(x_{i}^{-}(z)\right)=1 \otimes x_{i}^{-}(z)+x_{i}^{-}\left(z q^{c_{2}}\right) \otimes \psi_{i}\left(z q^{\frac{c_{2}}{2}}\right), \\
& \Delta\left(\varphi_{i}(z)\right)=\varphi_{i}\left(z q^{-\frac{c_{2}}{2}}\right) \otimes \varphi_{i}\left(z q^{\frac{c_{1}}{2}}\right), \\
& \Delta\left(\phi_{i}(z)\right)=\psi_{i}\left(z q^{\frac{c_{2}}{2}}\right) \otimes \psi_{i}\left(z q^{-\frac{c_{1}}{2}}\right),
\end{aligned}
$$

where $c_{1}$ means the action of the center on the first component and $c_{2}$ means the action of the center on the second component.

Counit $\varepsilon$

$$
\begin{array}{cl}
\varepsilon\left(q^{c}\right)=1 & \varepsilon\left(\varphi_{i}(z)\right)=\varepsilon\left(\phi_{i}(z)\right)=1 \\
& \varepsilon\left(x_{i}^{ \pm}(z)\right)=0 .
\end{array}
$$

Antipode a
(0) $a\left(q^{c}\right)=q^{-c}$,
(1) $a\left(x_{i}^{+}(z)\right)=-\varphi_{i}\left(z q^{-\frac{c}{2}}\right)^{-1} x_{i}^{+}\left(z q^{-c}\right)$,
(2) $a\left(x_{i}^{-}(z)\right)=-x_{i}^{-}\left(z q^{-c}\right) \psi_{i}\left(z q^{-\frac{c}{2}}\right)^{-1}$,
(3) $a\left(\varphi_{i}(z)\right)=\varphi_{i}(z)^{-1}$,
(4) $a\left(\phi_{i}(z)\right)=\psi_{i}(z)^{-1}$. 
We will give the following example to explain the comultiplication.

$$
\varphi_{i}\left(z q^{\left.\frac{c_{1}}{2}\right)} \otimes x_{i}^{+}\left(z q^{c_{1}}\right)=\sum_{l_{1} \in-\mathbf{z}_{\geq 0}, l_{2} \in \mathbf{Z}_{\geq 0}} z^{-\left(l_{1}+l_{2}\right)} q^{-\left(\frac{l_{1}}{2}+l_{2}\right)(c \otimes 1)} \varphi_{i}\left(l_{1}\right) \otimes x_{i}^{+}\left(l_{2}\right) .\right.
$$

It is clear that the comultiplication structure requires certain completion on the tensor space. For certain representations, such as the 2-dimensional representations of $U_{q}(\hat{\mathfrak{S} l}(2))$ at a special value, this comultiplication may not be well-defined. Nevertheless, this comultiplication is well-defined if one of the factor is a highest weight representation, because the action of the operator as a coefficient of $z^{m}$ of the currents operators on any element of such a module are zero if $m$ is small enough. The explicit proof for the theorem above for the case of $U_{q}(\hat{\mathfrak{b} l}(2))$ is given in [DI].

We will start with the Frenkel-Jing construction of level 1 representation of $U_{q}(\hat{\mathfrak{g} l}(2))$ on the Fock space.

Consider an algebra generated by $\left\{a_{k} \mid k \in \mathbb{Z} \backslash\{0\}\right\}$ satisfying :

$$
\left[a_{k}, a_{l}\right]=\delta_{k+l, 0} \frac{[2 k][k]}{k} \text {. }
$$

We call it the Heisenberg algebra.

Let $\bar{Q}=\mathbf{Z} \alpha$ be the root lattice of $\mathfrak{g l}(2)$. Let us define a group algebra $\mathbb{C}(q)[\overline{\mathcal{P}}]$, where $\overline{\mathcal{P}}$ is the weight lattice of $\mathfrak{g} l(2)$. Let $\bar{\Lambda}_{1}$ be the fundamental weight of $\mathfrak{g l}(2)$ and $2 \bar{\Lambda}_{1}=\alpha$. Let $\bar{\Lambda}_{0}=0$.

Set

$$
\mathscr{F}_{i}:=\mathbf{C}(q)\left[a_{-k}\left(k \in \mathbf{Z}_{>0}\right)\right] \otimes \mathbf{C}(q)[\bar{Q}] e^{\bar{\Lambda}_{1}} .
$$

This gives the Fock space.

The action of operators $a_{k}, \partial_{\alpha}, e^{\alpha}(1 \leq j \leq N)$ is given by

$$
\begin{array}{ll}
a_{k} \cdot f \otimes e^{\beta}= \begin{cases}a_{k} f \otimes e^{\beta} & k<0 ; \\
{\left[a_{k}, f\right] \otimes e^{\beta}} & k>0,\end{cases} \\
\partial_{\alpha} \cdot f \otimes e^{\beta}=(\alpha, \beta) f \otimes e^{\beta} & \text { for } f \otimes e^{\beta} \in \mathcal{F}_{i}, \\
e^{\alpha} \cdot f \otimes e^{\beta}=f \otimes e^{\alpha+\beta} . &
\end{array}
$$

Theorem 3. The following action on $\mathscr{F}_{i}$ of $U_{q}(\hat{\mathfrak{S l}}(2))$ gives a level 1 highest weight representation with the i-th fundamental weight as highest weight.

$$
\begin{aligned}
x^{ \pm}(z) & \mapsto \exp \left[ \pm \sum_{k>0} \frac{a_{-k}}{[k]} q^{\mp \frac{1}{2} k} z^{k}\right] \exp \left[\mp \sum_{k>0} \frac{a_{k}}{[k]} q^{\mp \frac{1}{2} k} z^{-k}\right] e^{ \pm \alpha} z^{ \pm \partial_{\alpha}+1} \\
\varphi(z) & \mapsto \exp \left[-\left(q-q^{-1}\right) \sum_{k>0} a_{-k} z^{k}\right] q^{-\partial_{\alpha}} \\
\psi(z) & \mapsto \exp \left[\left(q-q^{-1}\right) \sum_{k>0} a_{k} z^{-k}\right] q^{\partial_{\alpha}}
\end{aligned}
$$

This implies that on $\mathscr{F}_{i}$ 


$$
\begin{aligned}
& x^{+}(z) x^{+}(w)=z^{2}\left(1-\frac{w}{z}\right)\left(1-\frac{w}{z q^{2}}\right): x^{+}(z) x^{+}(w): \\
& x^{-}(z) x^{-}(w)=z^{2}\left(1-\frac{w}{z}\right)\left(1-\frac{w q^{2}}{z}\right): x^{-}(z) x^{-}(w): \\
& x^{+}(z) \varphi(w)=q^{-2} \frac{1-\frac{w}{q^{5 / 2} z}}{1-\frac{q^{3 / 2} w}{z}}: \varphi(w) x^{+}(z):=\frac{1-\frac{w}{q^{5 / 2} z}}{1-\frac{q^{3 / 2} w}{z}} \varphi(w) x^{+}(z), \\
& \psi(w) x^{-}(z)=\frac{1-\frac{q^{5 / 2} z}{w}}{1-\frac{z}{q^{3 / 2} w}} \psi(w) x^{-}(z):=\frac{q^{5 / 2} z}{w} \\
& 1-\frac{z}{q^{3 / 2} w}
\end{aligned}
$$

Lemma 4. Set $F=\oplus_{i=0,1} \mathcal{F}_{i .}$. Any level $m$ integrable module is a submodule of $\otimes{ }^{m} F$.

It is clear, for the case of $\hat{\mathfrak{s l}}(2)$, we have that the correlation functions of $e(z) e(w)$ and $f(z) f(w)$ have no poles, which are always polynomials of $z, z^{-1}$, $w, w^{-1}$. By the correlation functions of an operator, we mean all the matrix coefficients of the operator. For the quantum case, the correlation functions might have poles. However, the position of poles are restricted. We have

Proposition 5. For any level $k \geq 1$ integrable module of $U_{q}(\hat{\mathfrak{g l}}(2))$, the correlation functions of $x^{+}(z) x^{+}(w)$ has at most poles at $w / z=q^{-2}$.

For the proof, set

$$
\Delta^{(0)}=1, \Delta^{(l)}=(\underbrace{1 \otimes \cdots \otimes 1}_{l-1} \otimes \Delta) \Delta^{(l-1)} .
$$

From the comultiplication formula, we know that on $\otimes^{k} F$ we have

$$
\begin{aligned}
& \Delta^{(k-1)}\left(x^{+}(z)\right)=\sum_{a=1}^{k} X_{a}^{+(k)}(z), \\
& X_{a}^{+(k)}(z)=\varphi\left(z q^{1 / 2}\right) \otimes \varphi\left(z q^{3 / 2}\right) \otimes \cdots \otimes \varphi\left(z q^{a-3 / 2}\right) \otimes x^{+}\left(z q^{a-1}\right) \otimes \overbrace{1 \otimes \cdots \otimes 1}^{k-a}
\end{aligned}
$$

Let $0<a<b \leq k$. The product $X_{a}^{+(k)}(z) X_{b}^{+(k)}(w)$ has a pole at $z=q^{2} w$ because it contains $x^{+}\left(z q^{a-1}\right) \varphi\left(w q^{a-1 / 2}\right)$. The other terms do not have any poles. Thus, with the lemma above, we complete the proof.

Similarly, we can show that

Proposition 6. For any level $k \geq 1$ integrable module of $U_{q}(\hat{\mathfrak{g l}}(2))$, the correlation functions of $x^{-}(z) x^{-}(w)$ has at most poles at $z q^{2}=w$. 
Now we state our main result.

Theorem 7. For any level $k \geq 1$ integrable module of $U_{q}(\hat{\mathfrak{s} l}(2))$, the correlation functions of $x^{+}\left(z_{1}\right) x^{+}\left(z_{2}\right) \cdots x^{+}\left(z_{k}\right) x^{+}\left(z_{k+1}\right)$ is zero if $z_{2} / z_{1}=z_{3} / z_{2}=$ $\cdots=z_{k+1} / z_{k}=q^{2}$.

We will prove that the correlation functions of

$$
X_{a_{1}}^{+(k)}\left(z_{1}\right) \cdots X_{a_{k+1}}^{+(k)}\left(z_{k+1}\right)
$$

is zero if $z_{2} / z_{1}=z_{3} / z_{2}=\cdots=z_{k+1} / z_{k}=q^{2}$.

Suppose that $a_{m}<a_{m+1}$ for some $m$. Then, the $a_{m}$-th tensor component of (5) contains $x^{+}\left(z_{m} q^{a_{m-1}}\right) \varphi\left(z_{m+1} q^{a_{m-1 / 2}}\right)$. From (3) we see that this product has a zero at $z_{m+1}=q^{2} z_{m}$. Similarly, if $a_{m}=a_{m+1}$ the product $x^{+}\left(z_{m} q^{a_{m-1}}\right) x^{+}\left(z_{m+1} q^{a_{m-1}}\right)$ has a zero at $z_{m+1}=q^{2} z_{m}$. Proposition 5 shows that no poles from other terms cancel these zeros at $z_{m+1}=q^{2} z_{m}$. Therefore, we conclude that (5) is zero if $z_{2} / z_{1}=z_{3} / z_{2}=\cdots=z_{m+1} / z_{m}=q^{2}$, unless $a_{m}>a_{m+1}$ for all $1 \leq m \leq k$. However, because $1 \leq a_{m} \leq k$, the last case never occurs. Thus we finish the proof.

Similarly we have

Theorem 8. For any level $k \geq 1$ integrable module of $U_{q}(\hat{\mathfrak{g l}}(2))$, the correlation functions of $x^{-}\left(z_{1}\right) x^{-}\left(z_{2}\right) \cdots x^{-}\left(z_{k}\right) x^{-}\left(z_{k+1}\right)$ is zero if $z_{1} / z_{2}=z_{2} / z_{3}=$ $\cdots=z_{k} / z_{k+1}=q^{2}$.

For the case of $U_{q}(\hat{\mathfrak{s l}}(n))$, we have the following results, which can be proved as the case of $U_{q}(\hat{\mathfrak{g} l}(2))$.

Proposition 9. For any level $k \geq 1$ integrable module of $U_{q}(\hat{\mathfrak{s} l}(n))$, the correlation functions of $x_{i}^{+}(z) x_{i}^{+}(w)$ has at most poles at $z q^{-2}=w$; and the correlation functions of $x_{i \pm 1}^{+}(z) x_{i}^{+}(w)$ has at most poles at $z q=w$. the correlation functions of $x_{i}^{-}(z) x_{i}^{-}(w)$ has at most poles at $z q^{2}=w$; the correlation functions of $x_{i \pm 1}^{-}(z) x_{i}^{-}(w)$ has at most poles at $z q^{-1}=w$.

The proof for this, we need to use Frenkel-Jing construction to construct the level 1 representations. We will omit it here, which can be found in [FJ]. However we will list the following formulas, which are the key point of the proof.

On the level 1 representation in $[\mathrm{FJ}]$, we have:

$$
x_{i}^{+}(z) x_{i}^{+}(w)=z^{2}\left(1-\frac{w}{z}\right)\left(1-\frac{w}{z q^{2}}\right): x_{i}^{+}(z) x_{i}^{+}(w):
$$




$$
\begin{aligned}
& x_{i}^{-}(z) x_{i}^{-}(w)=z^{2}\left(1-\frac{w}{z}\right)\left(1-\frac{w q^{2}}{2}\right): x_{i}^{-}(z) x_{i}^{-}(w): \\
& x_{i}^{+}(z) \varphi_{i}(w)=q^{-2} \frac{1-\frac{w}{q^{5 / 2} z}}{1-\frac{q^{3 / 2} w}{z}}: \varphi_{i}(w) x_{i}^{+}(z):=\frac{1-\frac{w}{q^{5 / 2} z}}{1-\frac{q^{3 / 2} w}{z}} \varphi_{i}(w) x_{i}^{+}(z), \\
& \psi_{i}(w) x_{i}^{-}(z)=\frac{1-\frac{q^{5 / 2} z}{w}}{1-\frac{z}{q^{3 / 2} w}}: \psi_{i}(w) x_{i}^{-}(z):=\frac{1-\frac{q^{5 / 2} z}{w}}{1-\frac{z}{q^{3 / 2} w}} x_{i}^{-}(z) \psi_{i}(w) .
\end{aligned}
$$

If $a_{i j}=-1$,

$$
\begin{aligned}
& x_{i}^{ \pm}(z) x_{i}^{ \pm}(w)=\frac{\left(z-w q^{ \pm 1}\right)}{\left(z-w q^{\mp 1}\right)}: x_{i}^{+}(z) x_{i}^{+}(w): \\
& x_{i}^{+}(z) \varphi_{j}(w)=\frac{1-\frac{q^{1 / 2} w}{z}}{1-\frac{w}{q^{3 / 2} z}}: \varphi_{j}(w) x_{i}^{+}(z):=q^{-1} \frac{1-\frac{q^{1 / 2} w}{z}}{1-\frac{w}{q^{3 / 2} z}} \varphi_{j}(w) x_{i}^{+}(z), \\
& \psi_{i}(z) x_{j}^{-}(w)=\frac{\frac{w}{q^{1 / 2} z}-1}{\frac{q^{3 / 2} w}{z}-1}: x_{j}^{-}(w) \psi_{i}(z):=q \frac{\frac{w}{z q^{1 / 2}}-1}{\frac{q^{3 / 2} w}{z}-1} x_{i}^{-}(w) \psi_{i}(z) .
\end{aligned}
$$

If $a_{i j}=0$,

$$
\begin{gathered}
x_{i}^{ \pm}(z) x_{j}^{ \pm}(w)=x_{j}^{ \pm}(w) x_{i}^{ \pm}(z)=: x_{i}^{+}(z) x_{i}^{+}(w):, \\
x_{i}^{+}(z) \varphi_{j}(w)=\varphi_{j}(w) x_{i}^{+}(z)=: \varphi_{i}(w) x_{i}^{+}(z):, \\
\psi_{i}(z) x_{j}^{-}(w)=x_{j}^{-}(w) \phi_{i}(z)=: \phi_{i}(w) x_{i}^{-}(z):
\end{gathered}
$$

Theorem 10. For any level $k \geq 1$ integrable module of $U_{q}(\hat{\mathfrak{g} l}(n))$, the correlation functions of $x_{a_{1}}^{+}\left(z_{1}\right) x_{a_{2}}^{+}\left(z_{2}\right) \cdots x_{a_{k}}^{+}\left(z_{k}\right) x_{a_{k+1}}^{+}\left(z_{k+1}\right)$ is zero if

(a) $a_{i}-a_{i+1}=0$ or \pm 1 ,

(b) $z_{a_{i}} / z_{a_{i+1}}=q$ for $a_{i}-a_{i+1}= \pm 1$,

(c) $z_{a_{i}} / z_{a_{i+1}}=q^{-2}$ for $a_{i}-a_{i+1}=0$,

(d) $z_{a_{i}} / z_{a_{j}} \neq q^{-1}$ for $a_{i}-a_{j}= \pm 1$ and $i<j$,

(e) $z_{a_{i}} / z_{a_{j}} \neq q^{2}$ for $a_{i}=a_{j}$ and $i<j$;

the correlation functions of $x_{a_{1}}^{-}\left(z_{1}\right) x_{a_{2}}^{-}\left(z_{2}\right) \cdots x_{a_{k}}^{-}\left(z_{k}\right) x_{a_{k+1}}^{-}\left(z_{k+1}\right)$ is zero if

(a) $a_{i}-a_{i+1}=0$ or \pm 1 ,

(b) $z_{a_{i}} / z_{a_{i+1}}=q^{-1}$ for $a_{i}-a_{i+1}= \pm 1$,

(c) $z_{a_{i}} / z_{a_{i+1}}=q^{2}$ for $a_{i}-a_{i+1}=0$ and

(d) $z_{a_{i}} / z_{a_{j}} \neq q$ for $a_{i}-a_{j}= \pm 1$ and $i<j$,

(e) $z_{a_{i}} / z_{a_{j}} \neq q^{-2}$ for $a_{i}=a_{j}$ and $i<j$.

In the classical case, the condition of integrability is used [FS] to build semi-infinite construction of the corresponding integrable representations, we expect that we can use the quantum condition of integrability to derive similar constructions, which may even help us to resolve certain difficulty in the classical case. 


\section{Acknowledgement}

The authors thank B. Feigin and A. Stoyanovsky for discussions. J.D. is supported by the grant Reward research (A) 08740020 from the Ministry of Education of Japan.

\section{References}

[DF] Ding, J. and Frenkel, I.B., Isomorphism of two realizations of quantum affine algebra $U_{q}(\hat{g l}(n)), C M P, 156$ (1993), 277-300.

[DI] Ding, J. and Iohara, K., Drinfeld comultiplication and vertex operators, RIMS-1091, Preprint.

[Dr1] Drinfeld, V.G., Hopf algebra and the quantum Yang-Baxter Equation, Dokl. Akad. Nauk. SSSR, 283 (1985), 1060-1064.

[Dr2] —, Quantum Groups, ICM Proceedings, New York, Berkeley, 1986, 798-820.

[Dr3] - New realization of Yangian and quantum affine algebra, Soviet Math. Dokl., 36 (1988), 212-216.

[FS] Feigin, B.L. and Stoyanovsky, A.V., Quasi-particle models for the representations of Lie algebras and the geometry of the flag manifold, RIMS-942, Preprint.

[FJ] Frenkel, I.B. and Jing, N., Vertex representations of quantum affine algebras, Proc. Natl. Acad. Sci., USA, 85 (1988), 9373-9377.

[JI] Jimbo, M., A $q$-difference analogue of $U(\mathrm{~g})$ and Yang-Baxter equation, Lett. Math. Phys., 10 (1985), 63-69.

[LP] Lepowsky, J. and Primc, M., Structure of standard modules for the affine Lie algebra $A_{1}^{(1)}$, Contemp. Math. 45, AMS, Providence, 1985. 